\title{
Current Understanding of Immunity Against Schistosomiasis: Impact on Vaccine and Drug Development
}

This article was published in the following Dove Press journal: Research and Reports in Tropical Medicine

\author{
Adebayo J Molehin (1D) ${ }^{1,2}$ \\ 'Department of Internal Medicine, \\ School of Medicine, Texas Tech \\ University Health Sciences Center, \\ Lubbock, TX 79430, USA; ${ }^{2}$ Center for \\ Tropical Medicine and Infectious \\ Diseases, School of Medicine, Texas Tech \\ University Health Sciences Center, \\ Lubbock, TX 79430, USA
}

\begin{abstract}
Schistosomiasis is a neglected tropical disease inflicting significant morbidity in humans worldwide. The disease is caused by infections with a parasitic trematode belonging to the genus Schistosoma. Over 250 million people are currently infected globally, with an estimated disability-adjusted life-years of 1.9 million attributed to the disease. Current understanding, based on several immunological studies using experimental and human models of schistosomiasis, reveals that complex immune mechanisms play off each other in the acquisition of immune resistance to infection/reinfection. Nevertheless, the precise characteristics of these responses, the specific antigens against which they are elicited, and how these responses are intricately regulated are still being investigated. What is apparent is that immunity to schistosome infections develops slowly and over a prolonged period of time, augmented by the death of adult worms occurring naturally or by praziquantel therapy. In this review, aspects of immunity to schistosomiasis, host-parasite interactions and their impact on schistosomiasis vaccine development are discussed.
\end{abstract}

Keywords: schistosomiasis, immunity, schistosomiasis vaccine development, resistance to reinfection

\section{Introduction}

Schistosomiasis is an ancient disease that has plagued humans since around 1500 BC. $^{1}$ It is a neglected tropical disease caused by infection with one of six geographically distinct parasitic blood flukes belonging to the genus Schistosoma. ${ }^{2,3}$ The three most clinically important species are Schistosoma mansoni and $S$. japonicum, causing hepatointestinal schistosomiasis, and S. haematobium, causing urinary schistosomiasis. Geographically, transmission of S. mansoni is localized to Latin American countries, parts of Africa and the Arabian Peninsula, while S. japonicum is endemic in the People's Republic of China, the Philippines and Indonesia, and S. haematobium infections occur mainly in Africa and in certain loci in the Arabian Peninsula. ${ }^{4-6}$ Although schistosomiasis is primarily endemic in tropical and subtropical regions of the world, active transmission has now been reported in locales previously known to be free of schistosomiasis. ${ }^{7,8}$

Globally, schistosomiasis currently impacts over one billion people, with over a quarter of those currently infected in 78 countries and more than 780 million people at risk of infection. ${ }^{9,10}$ Despite the relatively low mortality rate associated with schistosomiasis, totaling 290,000 deaths annually, the disease causes
Correspondence: Adebayo J Molehin Department of Internal Medicine, School of Medicine,Texas Tech University Health Sciences Center, Lubbock, TX 79430 USA

Tel + I (806) 7432639

Email adebayo.molehin@ttuhsc.edu 
significant morbidity in humans, particularly in subSaharan Africa, where most of the infections occur. ${ }^{3}$ Clinically, the symptoms of schistosomiasis are chronic and stealthy, manifesting as fevers, anemia, hepatosplenomegaly, genital lesions and irreversible fibrotic tissue damage. The burden of schistosomiasis is estimated at 1.9 million disability-adjusted life-years (DALYs), ${ }^{11}$ although the reported DALYs associated with the disease vary tremendously depending on the sources cited. ${ }^{12}$

The control of schistosomiasis has been nothing short of challenging to both policy makers and the research community. Despite the benefits of having achieved some success in morbidity reduction in certain regions through the implementation of concerted approaches that combine mass drug administration (MDA), intermediate snail host molluscicide, health education, and water sanitation and hygiene (WASH) programs, these approaches have not been hugely successful ${ }^{13,14}$ owing to pertinent logistical challenges, including poor MDA coverage, unabated reinfection rates in hyperendemic areas, shortfall in drug delivery and adherence, and lack of appropriate infrastructure. While experts in the field agree that vaccination in conjunction with current control measures represents our best hope of achieving elimination, the blatant reality is that no such vaccine is currently available for human use. ${ }^{2}$ A review covering key features of schistosome biology and the development of schistosomiasis vaccine was published in $2020 .^{2}$ This present review discusses what is currently known regarding immunity to schistosome infection and reinfection, and the implications for vaccine and drug development.

\section{Immunology and Host-Parasite Interactions in Schistosome Infection}

Because of the complexity of the schistosome developmental cycle, the immune systems of infected hosts have to contend with multiple life-cycle stages of the parasite, including invading cercariae, migrating schistosomula, adult worms and eggs deposited by adult female worms. Cumulatively, all these stages in the infected host express thousands of highly antigenic molecules capable of eliciting strong humoral and cellular immune responses. ${ }^{15,16}$ While an increase in some of these antigen-specific responses is observable during the chronic phase of infection, others appear to be heavily downregulated. ${ }^{15,17}$ With respect to the immunology of human schistosomiasis, three main areas have been the subject of research: immunodiagnostics, immunopathogenesis and resistance to schistosome infection. While the immunodiagnostic aspect focuses on characterizing which types of elicited immune responses are diagnostic of schistosome infection, ${ }^{18} \mathrm{immu}-$ nopathogenesis is focused primarily on host immune responses to antigens released by eggs trapped within the tissues of hosts. ${ }^{3,5,19}$ One very important consideration in these areas of research is the continuous exposure to schistosome antigens over a prolonged period of time owing to the chronic nature and endemicity of schistosomiasis, resulting in fluidic maturation of the immune response to different levels of antigen exposure.

\section{What We Have Learnt from Experimental Animal Studies}

The establishment and the use of the experimental animal models of schistosomiasis have undoubtedly provided invaluable knowledge on the biology of schistosomes as well as insights into host-parasite interactions as they relate to immunity and the strategies employed to manipulate these responses. ${ }^{15,20}$ In particular, the murine models of schistosome infection and disease have provided a significant framework for the design of human immunological studies. ${ }^{20,21}$ Evidence from mouse studies has shown that both specific humoral and cell-mediated immune responses are essential for resistance against schistosome infection. ${ }^{20,22}$ Specifically, efficacy studies using radiation-attenuated (RA) cercariae revealed that the protection observed in mice exposed to attenuated cercariae was primarily associated with interferon- $\gamma$ (IFN- $\gamma$ ) production and antibody production after multiple rounds of vaccination. ${ }^{20,22-25}$ In other studies, coadministration of interleukin-12 (IL-12) with RA vaccine resulted in almost sterile immunity linked to a high induction of IFN- $\gamma,{ }^{26-28}$ while the induction of IL-10 seemed to diminish resistance to reinfection. ${ }^{29}$ Furthermore, mice deficient in IL-10, IL12p40 and IL-13R $\alpha 2$ had progressive and accelerated lethal liver fibrosis following $S$. mansoni infection. $^{30}$ Studies using experimental semi-/nonpermissive hosts, such as rats and rhesus macaques, have also shown that resistance to schistosome infections is almost entirely antibody dependent. ${ }^{31,32}$

Based on the success of RA cercariae vaccination, several candidate antigens were identified based on reactivity of cells and sera from immunized mice. The results from testing these candidate antigens were less promising 
but provided the premise for two vaccine candidates: recombinant $28-\mathrm{kDa}$ glutathione S-transferase of Schistosoma haematobium (rSh28GST) ${ }^{33-35}$ and $14-\mathrm{kDa}$ fatty acid-binding protein from Schistosoma mansoni (Sm14), ${ }^{36-40}$ which have successfully completed phase III and phase IIa trials, respectively. The development of other candidate antigens has focused primarily on identifying key molecules that play crucial roles in parasite survival, such as membrane biogenesis/renewal, nutrient uptake and immune evasion strategies. Some of the vaccines from these categories have been shown to also have some therapeutic properties, ${ }^{41}$ possibly by interfering with the adult worm evasion mechanisms and thereby making them susceptible to the host effector systems. ${ }^{15}$

During the chronic phase of schistosomiasis, granulomatous reactions form around the host trap eggs and their secreted egg antigens (SEAs), constituting the host defense against tissue attack/damage. The immunopathophysiology of schistosomiasis has been reviewed elsewhere. ${ }^{5,42}$ Since the granulomas themselves could be detrimental because of progression to liver fibrosis, studies have shown that infected mice develop effective anti-SEA immune responses (granuloma formation). ${ }^{42,43}$ Studies using gene knockout mice have also provided invaluable insights into the role of the host immune response to worm development and granuloma formation. ${ }^{44-46}$ The acute stage of infection in mice is mostly associated with a Th1type immune response which is dramatically shifted to a Th2-dependent response at the onset of oviposition. This immunological shift is thought to be caused by the interaction of certain carbohydrate epitopes from the egg antigens with the host dendritic cells. ${ }^{4,48}$ Although the upregulation of a Th2 cytokine IL-13 seemed to promote and exacerbate liver fibrosis, ${ }^{49}$ the complete abrogation of Th2 responses results in rapid fatality caused by uncontrolled severe tissue damage in IL-4-deficient mice infected with $S$. mansoni. ${ }^{50-52}$ In another study, the interruption of IL-4Ra-mediated signaling led to severe disease and fatality. ${ }^{53}$ While a Th1-type response is beneficial during the acute stages of schistosomiasis, an uncontrolled polarization toward the Th1 response, as shown in IL-10-/ IL-4-deficient mice, is lethally harmful to the host. ${ }^{54}$ In chronically infected patients, low levels of IL-5 and elevated levels of Th1-dependent cytokines are associated with severity of hepatosplenic schistosomiasis. ${ }^{55}$ Studies in mice have also demonstrated that the Th17-type immune response, particularly IL-17 production, promotes liver damage. ${ }^{56} \mathrm{IL}-12 \mathrm{p} 40^{-/-}$mice, deficient in IL-12 or IL-
23 production, showed significantly lower pathology associated with considerably low secretion of IFN- $\gamma$ and IL-17 by lymphoid cells stimulated with schistosome SEAs. In contrast, IL-12p35 ${ }^{-/-}$mice producing IL-23 and not IL-12 developed severe granulomas linked to high levels of IL$17 .^{56}$ Follicular helper T cells and Th9 cells have also been implicated in promoting egg-induced hepatic granulomas and fibrosis. ${ }^{57,58}$ Thus, Th2 immunity functions like a twoedged sword, on one hand protecting the host against excessive granulomatous inflammation, and on the other, causing host immune-dependent liver damage. A balance between Th1 and Th2 responses may therefore be necessary to prevent severe pathology. ${ }^{59}$ A schematic summarizing some of the key components of the host immune response during schistosome infection is shown in Figure 1.

The murine models of schistosomiasis have definitely contributed immensely to our understanding of the immunology and immunopathology of schistosomiasis; however, these models are not without their limitations and flaws. One of the major limitations of the mouse model is the low level of recovered adult worms following experimental challenge with penetrating cercariae. ${ }^{28}$ In addition, since infected mice do not live long enough after primary schistosome infection, they cannot be used in studies on immune correlates of reinfection resistance.

\section{What We Have Learnt from Human Studies}

Human epidemiological studies have provided useful information on host-schistosome interactions that complement the data from experimental animal studies. When studying the immunology of human schistosomiasis, it is pertinent to bear in mind that there are a number of unique facets to the host-parasite relationships involving the different life stages exposed to the host immune system. What is very clear is that there are overriding differences between the host immune response profiles against antigens derived from worms and those derived from eggs, regardless of the endemic population sampled. ${ }^{60}$ While most studies report robust immune responses to egg antigens early on, which then taper off as infections proceed into the chronic stage, induced responses to soluble worm antigen preparations are sustained throughout the chronic stage of the disease. ${ }^{61-63}$

Because of the heterogeneity of human populations, the status of the individuals being studied is also crucial. For 


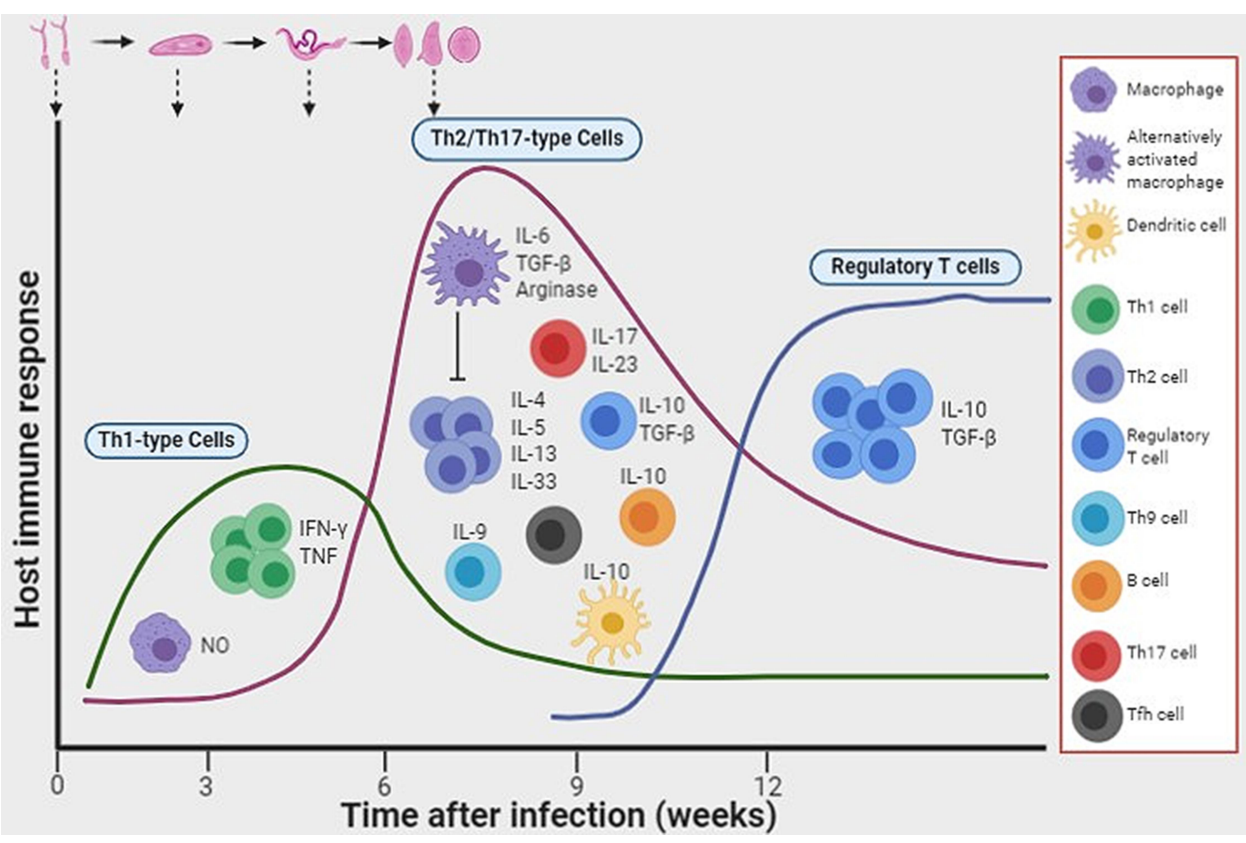

Figure I Induction of host immune responses after infection with schistosomes. Following infection with schistosomes, the early immune response that develops is a T helper I (ThI)-dependent cellular response. As the worms mature into adults and the females start to lay eggs, there is an increased production of interleukin- I0 (IL-I0) by dendritic cells, and a Th2 response ensues. In addition, B cells produce IL- 10 in response to antigens derived from eggs and adult worms. Populations of regulatory T cells and alternatively activated macrophages also develop. Image created with BioRender (app.biorender.com)

Abbreviations: NO, nitric oxide; IFN- $\gamma$, interferon- $\gamma$; TNF, tumor necrosis factor; TGF- $\beta$, transforming growth factor- $\beta$; Tfh, follicular helper T cells.

instance, it is important to know whether the individuals are currently uninfected or infected, and for how long, ${ }^{64,65}$ whether their mothers were infected during pregnancy ${ }^{66}$ and whether they have been treated with praziquantel (PZQ). ${ }^{67,68}$ All of the aforementioned factors, including many other scenarios, will determine a person's immune status at the time of study. Therefore, when interpreting these human population studies, one should always remember that the study of human immune responses to schistosomiasis is almost exclusively based on serum antibodies, cytokines and/or the responsiveness of peripheral blood mononuclear cells. It is possible that these sources may not be a true representation of what is happening within the immune microenvironment of a granuloma or around migrating schistosomula. Nonetheless, these are the specimens that are readily available, except in very rare circumstances when tissue becomes available through biopsy or autopsy. Regardless of these limitations, several aspects of the immunology of human schistosomiasis have been successfully characterized.

\section{Immunity to Reinfection in Humans}

The possibility of humans acquiring immune resistance to schistosome reinfection has long been a subject of discussion. ${ }^{69}$ Clear evidence now indicates that protective immunity does develop in people living in endemic areas, however very slowly. ${ }^{17,19,70}$ The hypothesis that the acquired schistosomiasis resistance observed in humans is linked to the stimulation of host protective immune responses to antigens released as a result of worm death is supported by many studies. ${ }^{71-74}$ Regardless of the geographic setting, epidemiological and immune correlate data obtained from schistosome-endemic regions indicate an age-dependent acquisition of resistance to reinfection. Children under the age of eleven are generally more susceptible to infection and reinfection in endemic areas than their adult counterparts, partly as a result of their history of exposure and infection intensity. ${ }^{3,75-77}$ Identifying immune "signatures" that correlate with protection is a key process in understanding resistance to schistosome infection and/or reinfection.

As already discussed, resistance to schistosomiasis in mice is associated primarily with Th1-type immune responses (see "What We Have Learnt from Experimental Animal Studies"). In contrast, in humans, Th2-type responses (although not exclusively) have been repeatedly associated with protection against reinfection. Multiple lines of evidence indicate that the production of parasite-specific IgE, eosinophils and cytokines such as IL-5 and IL-4 is required for resistance to reinfection, ${ }^{78-84}$ and that soluble IgE receptors and/or those expressed on eosinophils and B cells are also associated with 
resistance to reinfection. ${ }^{85}$ IL-33, a member of the IL-1 family of cytokines, has been associated with Th2-dependent protective immune response against helminth infections. ${ }^{86}$ A 2018 study showed an inverse correlation between plasma IL-33 levels and schistosome pathology in children living in an endemic area. ${ }^{87}$ In contrast to the protective effects of higher schistosome-specific $\mathrm{IgE}$ production, induction of $\mathrm{IgG}_{4}$ is linked to susceptibility to infection, potentially negating the protective effects of other antibody types, including $\operatorname{IgE} .{ }^{82,88}$ Following PZQ therapy, the level of parasite-specific $\mathrm{IgG}_{4}$ in infected adults decreases while $\mathrm{IgE}$ maintains pretreatment levels or sometimes increases, whereas the ratio of $\mathrm{IgE} / \mathrm{IgG}_{4}$ levels in children following treatment seems to be lowered. ${ }^{15}$ The production of $\mathrm{IgG}_{4}$ in humans by IL-10-producing regulatory $\mathrm{B}$ cells ${ }^{88}$ is consistent with the finding that IL-10 production in mice negates the development of resistance to reinfection. ${ }^{29}$

The general hypothesis is that the death of adult worms, either occurring naturally or following PZQ treatment, results in the release of parasite immunogens which cross-react with antigens from migrating larvae and consequently stimulate protective $\operatorname{IgE}$ responses. The implication is that the greater the exposure, the more rigorous the protective immune response generated. ${ }^{17}$ This hypothesis provides a plausible explanation as to why children are more susceptible to infection and reinfection while adults who have had many cycles of exposure and worm death develop resistance, albeit over a long period of time. ${ }^{5}$ Although IgE production appears to be critical to the development of resistance to schistosome reinfection in humans, developing and/or deploying a vaccine based on IgE is highly unlikely owing to documented health and safety issues relating to hypersensitivity reactions. ${ }^{12,89,90}$ Therefore, care should be taken with regard to how we mine and utilize the immune correlate findings from chronically infected individuals, particularly those with acquired resistance following PZQ treatment. Although ongoing schistosomiasis control programs do not feature vaccines, it is of great importance that research in this area intensifies as genuine progress toward schistosomiasis elimination and possible eradication can only be achieved through vaccination. ${ }^{2}$

\section{Human Challenge Model: A New Paradigm}

A team of researchers led by Dr. Meta Roestenberg, from Leiden University Medical Center in the Netherlands, pioneered the first human challenge model of schistosomiasis. These scientists performed the first controlled human $S$. mansoni infection using male cercariae in order to assess infectivity and possible adverse events. ${ }^{91}$ The results showed that all naïve volunteers recruited for the study showed IgM and $\mathrm{IgG}_{1}$ seroconversion, as well as specific $\mathrm{CD}^{+} \mathrm{T}$ celldependent cytokines. ${ }^{91}$ Another clinical trial focusing on single-sex female controlled human $S$. mansoni infection is currently underway (https://clinicaltrials.gov/ct2/show/ NCT04269915). In another study using human skin explants, Winkel et al showed that penetrating S. mansoni cercariae induce regulatory immune responses characterized by the production of IL-10 and pro-inflammatory IL-6. ${ }^{92}$ There has also been a growing interest in the use of controlled human infection (CHI) models. ${ }^{93-96}$ Implementation of the CHI schistosome model (CHI-S) in the context of testing vaccine candidates would undoubtedly provide valuable information on early protective efficacy as well as data on vaccinemediated immune correlates of protection.

\section{Schistosomiasis Vaccines: Target Profiles, Recent Advances and Challenges}

Historically, vaccination has been the most effective approach for preventing diseases caused by infectious pathogens. ${ }^{12,97}$ Of the hundreds of candidate antigens that have been identified and tested in various animal models of schistosomiasis, only three have made it into clinical trials to date. Phase II and III clinical trials in Africa have already been concluded for $S$. haematobium 28-kDa glutathione S-transferase (rSh28GST), ${ }^{34,98}$ while S. mansoni $14-\mathrm{kDa}$ fatty acid-binding protein ( $\mathrm{Sm} 14)$ is currently in phase IIb clinical trials, ${ }^{36,99}$ and a phase $\mathrm{Ib}$ trial is underway for $S$. mansoni tetraspanin, a $9-\mathrm{kDa}$ surface antigen (Sm-TSP-2). ${ }^{100,101}$ Details on the immunogenicity, tolerability and/or efficacy of these candidates have been reviewed elsewhere. ${ }^{2}$ Another candidate antigen, the large subunit of S. mansoni calpain (Sm-p80), has been approved for human clinical trials which are scheduled to begin in $2021 .{ }^{102,103}$

Despite the considerable research efforts that have been devoted to the development of a schistosomiasis vaccine, progress toward identifying more promising candidates or actualizing the deployment of a licensed vaccine has been very slow. Reasons for this slow progress include the complexity of the parasite life cycle, parasite immune evasion strategies and limited knowledge on immune correlates of protection, to mention but a few. In order to continue an upward trajectory with respect to schistosomiasis vaccine development, there are 
still important questions that need to be addressed. For instance, what is (or should be) the ideal target profile of an effective schistosomiasis vaccine? In two separate meetings organized by the Bill and Melinda Gates Foundation and the National Institute of Allergy and Infectious Diseases, experts in the field came up with the ideal target profile (preferred product characteristics [PPC]) for a schistosomiasis vaccine. The general consensus from these meetings was that an ideal vaccine should reduce adult schistosome numbers and egg excretion by at least $75 \%$. $^{12,104,105}$ The advent of technologies to measure schistosome circulating anodic antigens (CAAs) ${ }^{106}$ and/or circulating cathodic antigens (CCAs) ${ }^{107}$ in serum or urine samples from infected individuals has made it possible to quantify worm burden in humans instead of just relying on fecal egg output, thereby providing valuable data on vaccine efficacy. In addition, other questions regarding how the vaccine should be tested, dosage, choice of adjuvant and frequency of administration need to be tackled. Even at the point when a candidate antigen is ready for clinical trials, the design and execution of the trials pose their own challenges. The regulatory requirements and the substantial cost required to conduct clinical trials are also worth mentioning. In spite of these seemingly unsurmountable challenges facing schistosomiasis vaccine development, the long-term potential public health benefits of deploying an effective vaccine make the continued development efforts worthwhile.

\section{Concluding Remarks}

There is still a lot to understand about the complexity of the host-schistosome relationship. As far as this hostschistosome interplay is concerned, it is imperative to always bear in mind that there are multiple and complex responses at play whether resistance to infection and/or reinfection or the immunopathology of schistosomiasis is being discussed. Despite the advances in technologies and their associated benefits, it is also useful to recognize that more tools and new approaches are still needed if we are to expand our horizon and fully understand this complex interplay. The advent of the CHI-S model ${ }^{91}$ and tools to manipulate parasites in controlled studies are now providing platforms for us to start generating more proof to complement correlation studies.

Finally, several key questions still need to be considered. What mechanism(s) are involved in schistosomula killing? What specific factors are employed by schistosomes to survive in their immunological hostile environment? Are the immune responses induced in a newborn from an infected mother protective or regulatory? In-depth correlative studies of various immune responses in addition to documented cases of chronically infected individuals and the development of resistance to reinfection in treated individuals will undoubtedly provide some useful answers.

\section{Disclosure}

The author reports no conflicts of interest for this work.

\section{References}

1. El-Khoby T, Galal N, Fenwick A, et al. The epidemiology of schistosomiasis in Egypt: summary findings in nine governorates. Am J Trop Med Hyg. 2000;62(2 Suppl):88-99. doi:10.4269/ ajtmh.2000.62.88

2. Molehin AJ. Schistosomiasis vaccine development: update on human clinical trials. J Biomed Sci. 2020;27(1):28. doi:10.1186/ s12929-020-0621-y

3. McManus DP, Dunne DW, Sacko M, Utzinger J, Vennervald BJ, Zhou XN. Schistosomiasis. Nat Rev Dis Primers. 2018;4(1):13. doi:10.1038/s41572-018-0013-8

4. Colley DG, Bustinduy AL, Secor WE, King CH. Human schistosomiasis. Lancet. 2014;383(9936):2253-2264. doi:10.1016/S0140-6736(13)61949-2

5. McManus DP, Bergquist R, Cai P, Ranasinghe S, Tebeje BM, You H. Schistosomiasis-from immunopathology to vaccines. Semin Immunopathol. 2020;42(3):355-371. doi:10.1007/s00281020-00789-x

6. Ross AG, Bartley PB, Sleigh AC, et al. Schistosomiasis. $N$ Engl J Med. 2002;346(16):1212-1220. doi:10.1056/NEJMra012396

7. Boissier J, Grech-Angelini S, Webster BL, et al. Outbreak of urogenital schistosomiasis in Corsica (France): an epidemiological case study. Lancet Infect Dis. 2016;16(8):971-979. doi:10.1016/S1473-3099(16)00175-4

8. Pennisi E. Hybridization may give some parasites a leg up. Science. 2018;361(6405):832-833. doi:10.1126/ science.361.6405.832

9. Hotez PJ, Alvarado M, Basanez MG, et al. The global burden of disease study 2010: interpretation and implications for the neglected tropical diseases. PLoS Negl Trop Dis. 2014;8(7): e2865. doi:10.1371/journal.pntd.0002865

10. Toor J, Alsallaq R, Truscott JE, et al. Are we on our way to achieving the 2020 goals for schistosomiasis morbidity control using current World Health Organization Guidelines? Clin Infect Dis. 2018;66(suppl_4):S245-S52. doi:10.1093/cid/ciy001

11. GBD 2016 DALYs and HALE Collaborators. Global, regional, and national disability-adjusted life-years (DALYs) for 333 diseases and injuries and healthy life expectancy (HALE) for 195 countries and territories, 1990-2016: a systematic analysis for the Global Burden of Disease Study 2016. Lancet. 2017;390 (10100):1260-1344. Doi:10.1016/S0140-6736(17)32130-X.

12. Molehin AJ, Rojo JU, Siddiqui SZ, Gray SA, Carter D, Siddiqui AA. Development of a schistosomiasis vaccine. Expert Rev Vaccines. 2016;15(5):619-627. doi:10.1586/ 14760584.2016.1131127

13. McManus DP. Defeating schistosomiasis. $N$ Engl $J$ Med. 2019;381(26):2567-2568. doi:10.1056/NEJMe1913771

14. Campbell SJ, Biritwum NK, Woods G, Velleman Y, Fleming F, Stothard JR. Tailoring Water, Sanitation, and Hygiene (WASH) targets for soil-transmitted helminthiasis and schistosomiasis control. Trends Parasitol. 2018;34(1):53-63. doi:10.1016/j. pt.2017.09.004 
15. Colley DG, Secor WE. Immunology of human schistosomiasis. Parasite Immunol. 2014;36(8):347-357. doi:10.1111/pim. 12087

16. Hokke CH, Fitzpatrick JM, Hoffmann KF. Integrating transcriptome, proteome and glycome analyses of Schistosoma biology. Trends Parasitol. 2007;23(4):165-174. doi:10.1016/j.pt.2007.02.007

17. Fitzsimmons CM, Jones FM, Pinot de Moira A, et al. Progressive cross-reactivity in IgE responses: an explanation for the slow development of human immunity to schistosomiasis? Infect Immun. 2012;80(12):4264-4270. doi:10.1128/IAI.00641-12

18. Weerakoon KG, Gobert GN, Cai P, McManus DP. Advances in the diagnosis of human schistosomiasis. Clin Microbiol Rev. 2015;28(4):939-967. doi:10.1128/CMR.00137-14

19. Gryseels B, Polman K, Clerinx J, Kestens L. Human schistosomiasis. Lancet. 2006;368(9541):1106-1118. doi:10.1016/S0140-6736(06)69440-3

20. Pearce EJ, MacDonald AS. The immunobiology of schistosomiasis. Nat Rev Immunol. 2002;2(7):499-511. doi:10.1038/nri843

21. Tebeje BM, Harvie M, You H, Rivera V, McManus DP. T cell-mediated immunity in CBA mice during Schistosoma japonicum infection. Exp Parasitol. 2019;204:107725. doi:10.1016/j. exppara.2019.107725

22. Jankovic D, Wynn TA, Kullberg MC, et al. Optimal vaccination against Schistosoma mansoni requires the induction of both $\mathrm{B}$ cell- and IFN-gamma-dependent effector mechanisms. J Immunol. 1999;162(1):345-351.

23. Hewitson JP, Hamblin PA, Mountford AP. Immunity induced by the radiation-attenuated schistosome vaccine. Parasite Immunol. 2005;27(7-8):271-280. doi:10.1111/j.1365-3024.2005.00764.x

24. Sher A, Hieny S, James SL, Asofsky R. Mechanisms of protective immunity against Schistosoma mansoni infection in mice vaccinated with irradiated cercariae. II. Analysis of immunity in hosts deficient in T lymphocytes, B lymphocytes, or complement. J Immunol. 1982;128(4):1880-1884.

25. Aitken R, Coulson PS, Wilson RA. Pulmonary leukocytic responses are linked to the acquired immunity of mice vaccinated with irradiated cercariae of Schistosoma mansoni. J Immunol. 1988;140(10):3573-3579.

26. Wilson RA, Coulson PS. Immune effector mechanisms against schistosomiasis: looking for a chink in the parasite's armour. Trends Parasitol. 2009;25(9):423-431. doi:10.1016/j. pt.2009.05.011

27. Wilson RA, Coulson PS, Mountford AP. Immune responses to the radiation-attenuated schistosome vaccine: what can we learn from knock-out mice? Immunol Lett. 1999;65(1-2):117-123. doi:10.1016/s0165-2478(98)00134-5

28. Wilson RA, Li XH, Castro-Borges W. Do schistosome vaccine trials in mice have an intrinsic flaw that generates spurious protection data? Parasit Vectors. 2016;9(1):89. doi:10.1186/ s13071-016-1369-9

29. Wilson MS, Cheever AW, White SD, Thompson RW, Wynn TA, Pearce EJ. IL-10 blocks the development of resistance to re-infection with Schistosoma mansoni. PLoS Pathog. 2011;7 (8):e1002171. doi:10.1371/journal.ppat.1002171

30. Mentink-Kane MM, Cheever AW, Wilson MS, et al. Accelerated and progressive and lethal liver fibrosis in mice that lack interleukin (IL)-10, IL-12p40, and IL-13Ralpha2. Gastroenterology. 2011;141(6):2200-2209. doi:10.1053/j.gastro.2011.08.008

31. Knopf PM, Nutman TB, Reasoner JA. Schistosoma mansoni: resistance to reinfection in the rat. Exp Parasitol. 1977;41 (1):74-82. doi:10.1016/0014-4894(77)90131-x

32. Krautz-Peterson G, Debatis M, Tremblay JM, et al. Schistosoma mansoni infection of mice, rats and humans elicits a strong antibody response to a limited number of reduction-sensitive epitopes on five major tegumental membrane proteins. PLoS Negl Trop Dis. 2017;11(1):e0005306. doi:10.1371/journal.pntd.0005306
33. Riveau G, Schacht AM, Dompnier JP, et al. Safety and efficacy of the rSh28GST urinary schistosomiasis vaccine: a Phase 3 randomized, controlled trial in Senegalese children. PLoS Negl Trop Dis. 2018;12(12):e0006968. doi:10.1371/journal.pntd.0006968

34. Riveau G, Deplanque D, Remoue F, et al. Safety and immunogenicity of rSh28GST antigen in humans: phase 1 randomized clinical study of a vaccine candidate against urinary schistosomiasis. PLoS Negl Trop Dis. 2012;6(7):e1704. doi:10.1371/journal.pntd.0001704

35. Lane A, Boulanger D, Riveau G, Capron A, Wilson RA. Murine immune responses to Schistosoma haematobium and the vaccine candidate rSh28GST. Parasite Immunol. 1998;20(8):359-367.

36. Santini-Oliveira M, Coler RN, Parra J, et al. Schistosomiasis vaccine candidate Sm14/GLA-SE: phase 1 safety and immunogenicity clinical trial in healthy, male adults. Vaccine. 2016;34 (4):586-594. doi:10.1016/j.vaccine.2015.10.027

37. Tendler M, Almeida M, Simpson A. Development of the Brazilian anti schistosomiasis vaccine based on the recombinant fatty acid binding protein Sm14 plus GLA-SE adjuvant. Front Immunol. 2015;6:218. doi:10.3389/fimmu.2015.00218

38. Tendler M, Almeida MS, Vilar MM, Pinto PM, Limaverde-Sousa G. Current status of the Sm14/GLA-SE schistosomiasis vaccine: overcoming barriers and paradigms towards the first anti-parasitic human (itarian) vaccine. Trop Med Infect Dis. 2018;3(4). doi:10.3390/tropicalmed3040121

39. Tendler M, Brito CA, Vilar MM, et al. A Schistosoma mansoni fatty acid-binding protein, $\mathrm{Sm} 14$, is the potential basis of a dual-purpose anti-helminth vaccine. Proc Natl Acad Sci U S A. 1996;93(1):269-273. doi:10.1073/pnas.93.1.269

40. Thaumaturgo N, Vilar MM, Diogo CM, Edelenyi R, Tendler M. Preliminary analysis of Sm14 in distinct fractions of Schistosoma mansoni adult worm extract. Mem Inst Oswaldo Cruz. 2001;96 (Suppl):79-83. doi:10.1590/s0074-02762001000900011

41. Karmakar S, Zhang W, Ahmad G, et al. Use of an Sm-p80-based therapeutic vaccine to kill established adult schistosome parasites in chronically infected baboons. J Infect Dis. 2014;209 (12):1929-1940. doi:10.1093/infdis/jiu031

42. Schwartz C, Fallon PG. Schistosoma "eggs-iting" the host: granuloma formation and egg excretion. Front Immunol. 2018;9:2492. doi:10.3389/fimmu.2018.02492

43. Hams E, Aviello G, Fallon PG. The schistosoma granuloma: friend or foe? Front Immunol. 2013;4:89. doi:10.3389/fimmu.2013.00089

44. Jankovic D, Cheever AW, Kullberg MC, et al. CD4+ T cell-mediated granulomatous pathology in schistosomiasis is downregulated by a B cell-dependent mechanism requiring $\mathrm{Fc}$ receptor signaling. J Exp Med. 1998;187(4):619-629. doi:10.1084/jem.187.4.619

45. Ndlovu H, Brombacher F. Role of IL-4Ralpha during acute schistosomiasis in mice. Parasite Immunol. 2014;36(9):421-427. doi:10.1111/pim.12080

46. Souza AL, Souza PR, Pereira CA, et al. Experimental infection with Schistosoma mansoni in CCR5-deficient mice is associated with increased disease severity, as CCR5 plays a role in controlling granulomatous inflammation. Infect Immun. 2011;79 (4):1741-1749. doi:10.1128/IAI.00502-10

47. Fairfax K, Nascimento M, Huang SC, Everts B, Pearce EJ. Th2 responses in schistosomiasis. Semin Immunopathol. 2012;34 (6):863-871. doi:10.1007/s00281-012-0354-4

48. Everts B, Hussaarts L, Driessen NN, et al. Schistosome-derived omega-1 drives Th2 polarization by suppressing protein synthesis following internalization by the mannose receptor. J Exp Med. 2012;209(10):1753-67,S1. doi:10.1084/jem.20111381

49. Chiaramonte MG, Cheever AW, Malley JD, Donaldson DD, Wynn TA. Studies of murine schistosomiasis reveal interleukin-13 blockade as a treatment for established and progressive liver fibrosis. Hepatology. 2001;34(2):273-282. doi:10.1053/jhep.2001.26376 
50. La Flamme AC, Patton EA, Bauman B, Pearce EJ. IL-4 plays a crucial role in regulating oxidative damage in the liver during schistosomiasis. J Immunol. 2001;166(3):1903-1911. doi:10.4049/jimmunol.166.3.1903

51. Patton EA, Brunet LR, La Flamme AC, Pedras-Vasconcelos J, Kopf M, Pearce EJ. Severe schistosomiasis in the absence of interleukin-4 (IL-4) is IL-12 independent. Infect Immun. 2001;69(1):589-592. doi:10.1128/IAI.69.1.589-592.2001

52. Patton EA, La Flamme AC, Pedras-Vasoncelos JA, Pearce EJ. Central role for interleukin-4 in regulating nitric oxide-mediated inhibition of T-cell proliferation and gamma interferon production in schistosomiasis. Infect Immun. 2002;70(1):177-184. doi:10.1128/iai.70.1.177-184.2002

53. Nono JK, Ndlovu H, Aziz NA, Mpotje T, Hlaka L, Brombacher F. Host regulation of liver fibroproliferative pathology during experimental schistosomiasis via interleukin-4 receptor alpha. PLoS Negl Trop Dis. 2017;11(8):e0005861. doi:10.1371/journal. pntd. 0005861

54. Hoffmann KF, Cheever AW, Wynn TA. IL-10 and the dangers of immune polarization: excessive type 1 and type 2 cytokine responses induce distinct forms of lethal immunopathology in murine schistosomiasis. J Immunol. 2000;164(12):6406-6416. doi:10.4049/jimmunol.164.12.6406

55. Mwatha JK, Kimani G, Kamau T, et al. High levels of TNF, soluble TNF receptors, soluble ICAM-1, and IFN-gamma, but low levels of IL-5, are associated with hepatosplenic disease in human schistosomiasis mansoni. J Immunol. 1998;160(4):1992-1999.

56. Rutitzky LI, Lopes da Rosa JR, Stadecker MJ. Severe CD4 T cell-mediated immunopathology in murine schistosomiasis is dependent on IL-12p40 and correlates with high levels of IL-17. J Immunol. 2005;175(6):3920-3926. doi:10.4049/jimmunol.175.6.3920

57. Chen X, Yang X, Li Y, et al. Follicular helper T cells promote liver pathology in mice during Schistosoma japonicum infection. PLoS Pathog. 2014;10(5):e1004097. doi:10.1371/journal. ppat. 1004097

58. Li L, Xie H, Wang M, et al. Characteristics of IL-9 induced by Schistosoma japonicum infection in C57BL/6 mouse liver. Sci Rep. 2017;7(1):2343. doi:10.1038/s41598-017-02422-8

59. Peine M, Rausch S, Helmstetter C, et al. Stable T-bet(+)GATA-3(+) Th1/Th2 hybrid cells arise in vivo, can develop directly from naive precursors, and limit immunopathologic inflammation. PLoS Biol. 2013;11(8):e1001633. doi:10.1371/journal.pbio.1001633

60. Williams ME, Montenegro S, Domingues AL, et al. Leukocytes of patients with Schistosoma mansoni respond with a Th2 pattern of cytokine production to mitogen or egg antigens but with a Th0 pattern to worm antigens. J Infect Dis. 1994;170(4):946-954. doi:10.1093/infdis/170.4.946

61. Barsoum IS, Gamil FM, Al-Khafif MA, Ramzy RM, El Alamy MA, Colley DG. Immune responses and immunoregulation in relation to human schistosomiasis in Egypt. I. Effect of treatment on in vitro cellular responsiveness. Am J Trop Med Hyg. 1982;31(6):1181-1187. doi:10.4269/ajtmh.1982.31.1181

62. Caldas IR, Campi-Azevedo AC, Oliveira LF, Silveira AM, Oliveira RC, Gazzinelli G. Human schistosomiasis mansoni: immune responses during acute and chronic phases of the infection. Acta Trop. 2008;108(2-3):109-117. doi:10.1016/j. actatropica.2008.05.027

63. Colley DG, Garcia AA, Lambertucci JR, et al. Immune responses during human schistosomiasis. XII. Differential responsiveness in patients with hepatosplenic disease. Am J Trop Med Hyg. 1986;35 (4):793-802. doi:10.4269/ajtmh.1986.35.793

64. Naus CW, van Remoortere A, Ouma JH, et al. Specific antibody responses to three schistosome-related carbohydrate structures in recently exposed immigrants and established residents in an area of Schistosoma mansoni endemicity. Infect Immun. 2003;71 (10):5676-5681. doi:10.1128/iai.71.10.5676-5681.2003
65. Black CL, Mwinzi PN, Muok EM, et al. Influence of exposure history on the immunology and development of resistance to human Schistosomiasis mansoni. PLoS Negl Trop Dis. 2010;4 (3):e637. doi:10.1371/journal.pntd.0000637

66. Novato-Silva E, Gazzinelli G, Colley DG. Immune responses during human schistosomiasis mansoni. XVIII. Immunologic status of pregnant women and their neonates. Scand J Immunol. 1992;35(4):429-437. doi:10.1111/j.1365-3083.1992.tb02878.x

67. Black CL, Muok EM, Mwinzi PN, et al. Increases in levels of schistosome-specific immunoglobulin E and CD23(+) B cells in a cohort of Kenyan children undergoing repeated treatment and reinfection with Schistosoma mansoni. J Infect Dis. 2010;202 (3):399-405. doi:10.1086/653828

68. Watanabe K, Mwinzi PN, Black CL, et al. T regulatory cell levels decrease in people infected with Schistosoma mansoni on effective treatment. Am J Trop Med Hyg. 2007;77(4):676-682. doi:10.4269/ajtmh.2007.77.676

69. Warren KS. Regulation of the prevalence and intensity of schistosomiasis in man: immunology or ecology? J Infect Dis. 1973;127(5):595-609. doi:10.1093/infdis/127.5.595

70. Mutapi F, Billingsley PF, Secor WE. Infection and treatment immunizations for successful parasite vaccines. Trends Parasitol. 2013;29(3):135-141. doi:10.1016/j.pt.2013.01.003

71. Mutapi F, Ndhlovu PD, Hagan P, et al. Chemotherapy accelerates the development of acquired immune responses to Schistosoma haematobium infection. J Infect Dis. 1998;178(1):289-293. doi:10.1086/517456

72. Mutapi F, Ndhlovu PD, Hagan P, Woolhouse ME. A comparison of re-infection rates with Schistosoma haematobium following chemotherapy in areas with high and low levels of infection. Parasite Immunol. 1999;21(5):253-259. doi:10.1046/j.13653024.1999.00227.x

73. Pinot de Moira A, Jones FM, Wilson S, et al. Effects of treatment on $\mathrm{IgE}$ responses against parasite allergen-like proteins and immunity to reinfection in childhood schistosome and hookworm coinfections. Infect Immun. 2013;81(1):23-32. doi:10.1128/IAI.00748-12

74. Wilson S, Jones FM, Fofana HK, et al. Rapidly boosted Plasma IL-5 induced by treatment of human Schistosomiasis haematobium is dependent on antigen dose, IgE and eosinophils. PLoS Negl Trop Dis. 2013;7(3):e2149. doi:10.1371/journal.pntd.0002149

75. Mitchell KM, Mutapi F, Savill NJ, Woolhouse ME. Explaining observed infection and antibody age-profiles in populations with urogenital schistosomiasis. PLoS Comput Biol. 2011;7(10): e1002237. doi:10.1371/journal.pcbi.1002237

76. Wilson S, Jones FM, van Dam GJ, et al. Human Schistosoma haematobium antifecundity immunity is dependent on transmission intensity and associated with immunoglobulin G1 to worm-derived antigens. J Infect Dis. 2014;210(12):2009-2016. doi:10.1093/infdis/jiu374

77. Osakunor DNM, Woolhouse MEJ, Mutapi F, Raso G. Paediatric schistosomiasis: what we know and what we need to know. PLoS Negl Trop Dis. 2018;12(2):e0006144. doi:10.1371/journal. pntd.0006144

78. Dunne DW, Butterworth AE, Fulford AJ, et al. Immunity after treatment of human schistosomiasis: association between $\mathrm{IgE}$ antibodies to adult worm antigens and resistance to reinfection. Eur J Immunol. 1992;22(6):1483-1494. doi:10.1002/ eji.1830220622

79. Dunne DW, Butterworth AE, Fulford AJ, Ouma JH, Sturrock RF. Human IgE responses to Schistosoma mansoni and resistance to reinfection. Mem Inst Oswaldo Cruz. 1992;87(Suppl 4):99-103. doi:10.1590/s0074-02761992000800014

80. Hagan P, Blumenthal UJ, Dunn D, Simpson AJ, Wilkins HA. Human IgE, IgG4 and resistance to reinfection with Schistosoma haematobium. Nature. 1991;349(6306):243-245. doi:10.1038/ $349243 \mathrm{a} 0$ 
81. Satti MZ, Lind P, Vennervald BJ, Sulaiman SM, Daffalla AA, Ghalib HW. Specific immunoglobulin measurements related to exposure and resistance to Schistosoma mansoni infection in Sudanese canal cleaners. Clin Exp Immunol. 1996;106(1):45-54. doi:10.1046/j.1365-2249.1996.d01-810.x

82. Oliveira RR, Figueiredo JP, Cardoso LS, et al. Factors associated with resistance to Schistosoma mansoni infection in an endemic area of Bahia, Brazil. Am J Trop Med Hyg. 2012;86(2):296-305. doi:10.4269/ajtmh.2012.11-0204

83. Ganley-Leal LM, Mwinzi PN, Cetre-Sossah CB, et al. Correlation between eosinophils and protection against reinfection with Schistosoma mansoni and the effect of human immunodeficiency virus type 1 coinfection in humans. Infect Immun. 2006;74 (4):2169-2176. doi:10.1128/IAI.74.4.2169-2176.2006

84. Negrao-Correa D, Fittipaldi JF, Lambertucci JR, Teixeira MM, Antunes CM, Carneiro M. Association of Schistosoma mansoni-specific IgG and IgE antibody production and clinical schistosomiasis status in a rural area of Minas Gerais, Brazil. PLoS One. 2014;9(2):e88042. doi:10.1371/journal. pone. 0088042

85. Mwinzi PN, Ganley-Leal L, Black CL, Secor WE, Karanja DM, Colley DG. Circulating CD23+ B cell subset correlates with the development of resistance to Schistosoma mansoni reinfection in occupationally exposed adults who have undergone multiple treatments. J Infect Dis. 2009;199(2):272-279. doi:10.1086/595792

86. Liew FY, Pitman NI, McInnes IB. Disease-associated functions of IL-33: the new kid in the IL-1 family. Nat Rev Immunol. 2010;10 (2):103-110. doi:10.1038/nri2692

87. Nono JK, Kamdem SD, Netongo PM, et al. Schistosomiasis burden and its association with lower measles vaccine responses in school children from rural Cameroon. Front Immunol. 2018;9:2295. doi:10.3389/fimmu.2018.02295

88. van de Veen W, Stanic B, Yaman G, et al. IgG4 production is confined to human IL-10-producing regulatory B cells that suppress antigen-specific immune responses. $J$ Allergy Clin Immunol. 2013;131(4):1204-1212. doi:10.1016/j.jaci.2013.01.014

89. Diemert DJ, Bottazzi ME, Plieskatt J, Hotez PJ, Bethony JM. Lessons along the critical path: developing vaccines against human helminths. Trends Parasitol. 2018;34(9):747-758. doi:10.1016/j.pt.2018.07.005

90. Diemert DJ, Pinto AG, Freire J, et al. Generalized urticaria induced by the Na-ASP-2 hookworm vaccine: implications for the development of vaccines against helminths. J Allergy Clin Immunol. 2012;130(1):169-76e6. doi:10.1016/j.jaci.2012.0 4.027

91. Langenberg MCC, Hoogerwerf MA, Koopman JPR, et al. A controlled human Schistosoma mansoni infection model to advance novel drugs, vaccines and diagnostics. Nat Med. 2020;26(3):326-332. doi:10.1038/s41591-020-0759-x

92. Winkel BMF, Dalenberg MR, de Korne CM, et al. Early induction of human regulatory dermal antigen presenting cells by skin-penetrating schistosoma mansoni cercariae. Front Immunol. 2018;9:2510. doi:10.3389/fimmu.2018.02510

93. Bachmann A, Bruske E, Krumkamp R, et al. Controlled human malaria infection with Plasmodium falciparum demonstrates impact of naturally acquired immunity on virulence gene expression. PLoS Pathog. 2019;15(7):e1007906. doi:10.1371/ journal.ppat.1007906
94. Roestenberg M, Mo A, Kremsner PG, Yazdanbakhsh M Controlled human infections: a report from the controlled human infection models workshop, Leiden University Medical Centre 4-6 May 2016. Vaccine. 2017;35(51):7070-7076. doi:10.1016/j.vaccine.2017.10.092

95. Roestenberg M, Mordmuller B, Ockenhouse C, Mo A, Yazdanbakhsh M, Kremsner PG. The frontline of controlled human malaria infections: a report from the controlled human infection models Workshop in Leiden University Medical Centre 5 May 2016. Vaccine. 2017;35(51):7065-7069. doi:10.1016/j.vaccine.2017.10.093

96. Gordon SB, Rylance J, Luck A, et al. A framework for Controlled Human Infection Model (CHIM) studies in Malawi: report of a Wellcome Trust workshop on CHIM in low income countries held in Blantyre, Malawi. Wellcome Open Res. 2017;2:70. doi:10.12688/wellcomeopenres.12256.1

97. Greenwood B. The contribution of vaccination to global health: past, present and future. Philos Trans R Soc Lond B Biol Sci. 2014;369(1645):20130433. doi:10.1098/rstb.2013.0433

98. Boulanger D, Warter A, Sellin B, et al. Vaccine potential of a recombinant glutathione S-transferase cloned from Schistosoma haematobium in primates experimentally infected with an homologous challenge. Vaccine. 1999;17(4):319-326. doi:10.1016/s0264-410x(98)00202-3

99. Moser D, Tendler M, Griffiths G, Klinkert MQ. A 14-kDa Schistosoma mansoni polypeptide is homologous to a gene family of fatty acid binding proteins. J Biol Chem. 1991;266(13):8447-8454.

100. Keitel WA, Potter GE, Diemert D, et al. A phase 1 study of the safety, reactogenicity, and immunogenicity of a Schistosoma mansoni vaccine with or without glucopyranosyl lipid A aqueous formulation (GLA-AF) in healthy adults from a non-endemic area. Vaccine. 2019;37(43):6500-6509. doi:10.1016/j.vaccine.2019.08.075

101. Tran MH, Pearson MS, Bethony JM, et al. Tetraspanins on the surface of Schistosoma mansoni are protective antigens against schistosomiasis. Nat Med. 2006;12(7):835-840. doi:10.1038/nm1430

102. Siddiqui AA, Siddiqui SZ. Sm-p80-based schistosomiasis vaccine: preparation for human clinical trials. Trends Parasitol. 2017;33(3):194-201. doi:10.1016/j.pt.2016.10.010

103. Siddiqui AA, Zhou Y, Podesta RB, et al. Characterization of $\mathrm{Ca}(2$ + )-dependent neutral protease (calpain) from human blood flukes, Schistosoma mansoni. Biochim Biophys Acta. 1993;1181 (1):37-44. doi:10.1016/0925-4439(93)90087-h

104. Mo AX, Agosti JM, Walson JL, Hall BF, Gordon L. Schistosomiasis elimination strategies and potential role of a vaccine in achieving global health goals. Am J Trop Med Hyg. 2014;90(1):54-60. doi:10.4269/ajtmh.13-0467

105. Mo AX, Colley DG. Workshop report: schistosomiasis vaccine clinical development and product characteristics. Vaccine. 2016;34(8):995-1001. doi:10.1016/j.vaccine.2015.12.032

106. Sousa MS, van Dam GJ, Pinheiro MCC, et al. Performance of an ultra-sensitive assay targeting the Circulating Anodic Antigen (CAA) for detection of schistosoma mansoni infection in a low endemic area in Brazil. Front Immunol. 2019;10:682. doi:10.3389/fimmu.2019.00682

107. van Dam GJ, Wichers JH, Ferreira TM, Ghati D, van Amerongen A, Deelder AM. Diagnosis of schistosomiasis by reagent strip test for detection of circulating cathodic antigen. $J$ Clin Microbiol. 2004;42(12):5458-5461. doi:10.1128/ JCM.42.12.5458-5461.2004 


\section{Publish your work in this journal}

Research and Reports in Tropical Medicine is an international, peerreviewed, open access journal publishing original research, case reports, editorials, reviews and commentaries on all areas of tropical medicine, including: Diseases and medicine in tropical regions; Entomology; Epidemiology; Health economics issues; Infectious disease; Laboratory science and new technology in tropical medicine;
Parasitology; Public health medicine/health care policy in tropical regions; and Microbiology. The manuscript management system is completely online and includes a very quick and fair peer-review system. Visit http://www.dovepress.com/testimonials.php to read real quotes from published authors. 